\title{
El turismo rural como estrategia de desarrollo sostenible para el municipio de Pitalito - Corregimiento de Bruselas (Huila). Una propuesta
}

\section{Rural tourism as a strategy of sustainable development for the town of Pitalito - Township of Bruselas (Huila). A proposal}

\author{
Carlos Enrique Parra Rodríguez \\ Magister en Diseño, Gestión y Dirección de Proyectos \\ Instructor Contratista - Cíes Sena. Neiva \\ cparra@sena.edu.co \\ Hernando Gil Tovar \\ Magister en Administración \\ Universidad Surcolombiana. Neiva. Colombia \\ Jennifer Alexandra Javela \\ Egresada del Programa de Contaduría Pública. \\ Universidad Surcolombiana. Neiva \\ Indira Muñoz urbano Cerón \\ Egresada del Programa de Economía. \\ Universidad Surcolombiana. Neiva. Colombia \\ Maber Rengifo Gonzales \\ Especialista en Administración Financiera. \\ Universidad EAN. Egresada Sena Cies. Neiva
}

\section{Introducción}

El potencial en recursos naturales con el que cuenta el corregimiento de Bruselas del municipio de Pitalito, la acogida que ha tenido a nivel mundial el producto del café especial y las diferentes prácticas agrícolas de esta zona, han sido el pilar de nuestra investigación, en la cual pretendemos demostrar, el turismo rural como estrategia de desarrollo sostenible, el cual se convierte en un potencial enorme en el corregimiento de Bruselas (Pitalito) en el área de turismo y se puede convertir en un ejemplo para la oferta turística rural a nivel nacional e internacional.

Se determinó el aporte del turismo rural en el cambio del estado de desarrollo de los habitantes del municipio de Pitalito y especialmente en el corregimiento de Bruselas (Huila), bajo el enfoque de "desarrollo humano sostenible". Se elaboró una revisión de los conceptos: medio rural, turismo, turismo rural, desarrollo y sostenibilidad que permitió enmarcar la investigación. Con la Metodología de Sistemas Suaves adaptada de Checkland se construyó un modelo ideal de un turismo rural que contribuyera al "desarrollo humano sostenible".
En los análisis recientes del mundo rural y en la elaboración de propuestas orientadas a promover su desarrollo, se destacan elementos como la multifuncionalidad de la agricultura, los empleos y los ingresos no agrícolas y el territorio como eje de aproximación al análisis y a la acción. Todos estos conceptos, permiten enmarcar la presentación de turismo rural como alternativa para encontrar empresas rurales vinculadas principalmente con la actividad de las labores del campo.

El turismo rural se perfila como un tipo de actividad turística que ofrece al visitante la posibilidad de conocer aspectos de la cultura local y de aprender sobre prácticas tradicionales de cultivos, cosechas y procesamiento de productos agropecuarios, forestales y pesqueros, además de la artesanía y la gastronomía.

Por otra parte, el turismo rural se constituye en una opción de diversificación de las actividades agrícolas, con lo cual se benefician no solo los propietarios, sino también otros pobladores rurales que, por este medio tienen nuevas fuentes de empleo e ingresos y argumentos adicionales para permanecer en los espacios rurales en los que han nacido y se han criado. La importancia de la investigación radica en la 
necesidad de investigar el turismo rural como una estrategia de desarrollo sostenible, con el fin de motivar y capacitar a la población rural en la implementación de investigación dinamizadores que permitan el desarrollo económico y el conocimiento de las riquezas que posee Pitalito en especial el corregimiento de Bruselas.

El modelo se contrastó con lo encontrado en el Municipio, donde, a través de la misma metodología en conjunto con la "inmersión social" se caracterizó el sistema turístico del Corregimiento de Bruselas (Pitalito - Huila) en el momento de la investigación. Al realizar el análisis se evidenciaron conflictos originados por las cosmovisiones divergentes sobre el turismo rural en el territorio, de diferentes grupos de actores locales interesados o afectados por la actividad turística. Se encontró que en el municipio no existía un sistema de turismo rural claramente delimitado y que el modelo de turismo que se realizaba no estaba contribuyendo al desarrollo sostenible de sus habitantes de manera generalizada.

\section{Problema de investigación y su justificación}

La importancia de este tema radica en su población y en el auge que ha tenido el café especial en el mundo y en especial en el corregimiento de Bruselas municipio de Pitalito, en donde hay un grupo de personas quienes adelantan turismo rural y están permitiendo el aprovechamiento de los recursos naturales para atraer turistas y mostrar el enriquecimiento de la región a nivel de fauna y flora. Esto nos hizo reflexionar en cómo podríamos contribuir como estrategia de desarrollo sostenible para el corregimiento y su municipio, ayudando a dinamizar el sector rural desde la perspectiva de la auto sostenibilidad conservando el medio ambiente y teniendo en cuenta las características propias del lugar con base en teorías económicas, culturales, sociales, que admiten ámbitos y concepciones de aportes frente a la interacción social, la adaptación entre el individuo y su medio y otras de vital importancia que se encuentran descritas en el marco referencial, y son el sustento para analizar la necesidad de capacitación que tienen los habitantes del corregimiento de Bruselas interesados en realizar investigación donde el turismo rural se convierta en una alternativa de desarrollo autosostenible.

El área de influencia de cobertura de la investigación es el asiento del Macizo Colombiano y el anillo turístico del Huila, exactamente el Corregimiento de Bruselas ubicado por la vía a Mocoa, integrado por las veredas: el cedro, Montecristo, Villa Fátima, Cristalina, El encanto, El diamante, El mesón, Holanda, Campo bello, La palma, Cabuyal del cedro, Cabeceras, Cerritos, Guandinosa, Hacienda Bruselas, Bombonal, Miraflores, El palmito, Porvenir, la Esperanza, El pencil, El Carmen, Primavera, Esmeralda, Lomitas, Bruselas, Santafé, Las brisas, Kennedy, Alto de la Cruz, Puerto lleras y Normandía.

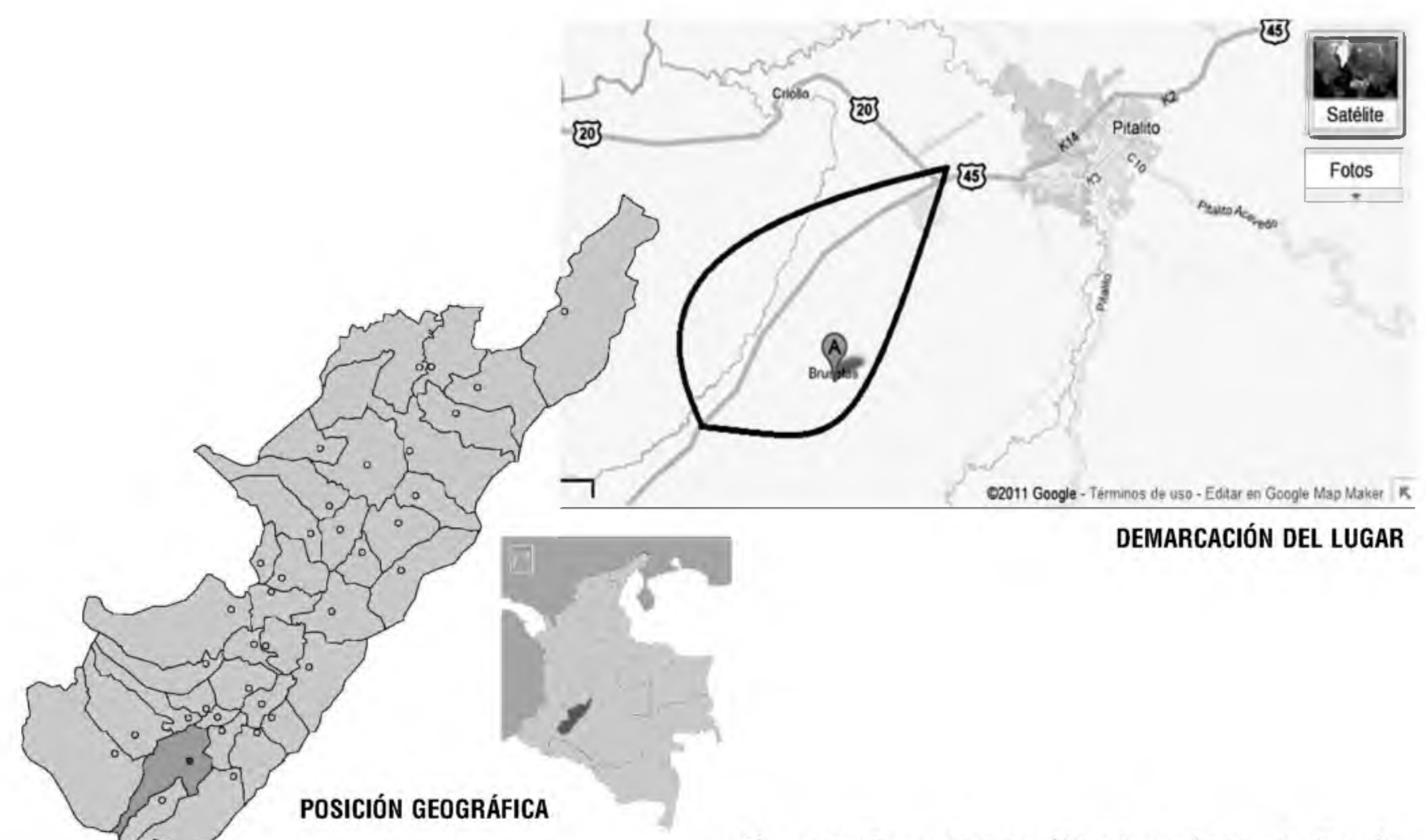

Figura 1. Ubicación geográfica de Pitalito según Google. 
En atención a lo anterior, esta proyecto gira en torno a un problema de investigación específico en un primer momento ¿Cómo lograr que el turismo rural sea una actividad conducente al desarrollo sostenible de la población rural del corregimiento de Bruselas (Pitalito)

La investigación busca lograr que el turismo sea una actividad conducente al desarrollo sostenible de la población rural en el territorio del corregimiento de Bruselas (Pitalito - Huila).

En nuestro país, debido a razones endógenas como el conflicto armado, ha provocado que el interés por el desarrollo del campo decrezca. Cada vez son más los campesinos que se adentran en las junglas de cemento porque consideran que el desarrollo se encuentra allí a través de múltiples oportunidades de trabajo y lo más importante, de seguridad.

Con el proyecto de investigación se hace una propuesta en donde el turismo sería el eje del desarrollo sostenible de la población rural en el territorio del corregimiento de Bruselas (Pitalito - Huila) y de ésta forma contribuir en la disminución de Insuficiencia de investigación dinamizadores de desarrollo auto sostenible desde el sector turístico. La investigación promueve el desarrollo local mediante diferentes líneas de trabajo una de ellas es brindar asesoramiento a las comunidades que tienen un gran potencial para comercializar productos y servicios turísticos, donde se busca convertir fincas rurales en lugares de descanso y sano esparcimiento que permitirá a los amantes de la naturaleza apreciar el entorno que los rodea así como adquirir variedad de productos y disfrutar de la gastronomía propia del lugar.

Por otra parte, el turismo rural se constituye en una opción de diversificación de las actividades agrícolas, con lo cual se benefician no solo los propietarios, sino también otros pobladores rurales que, por este medio tienen nuevas fuentes de empleo e ingresos y argumentos adicionales para permanecer en los espacios rurales en los que han nacido y han crecido.

El municipio de Pitalito atesora, en sus áreas rurales, un valioso patrimonio natural y cultural con potencial para el desarrollo turístico. Montes, cascadas, sierras, quebradas, así como la cercanía al parque arqueológico de san Agustín, hacen un atractivo para todos aquellos que llegan a esta zona con el fin de conocer, apreciar y disfrutar de nuestra herencia natural y cultural.

Por tal motivo es necesario brindar herramientas necesarias para motivar y capacitar a los habitantes de la región de Bruselas y quienes tengan la capacidad de elaborar investigación con visión empresarial, con el fin de convertir el turismo rural en una alternativa económica visible, para mejorar la calidad de vida de sus habitantes.

Los beneficiarios de la investigación son pobladores de comunidades rurales andinas cuyo sustento proviene principalmente de la actividad agrícola, familias que cuentan con un promedio de 6-8 personas abarcando generalmente tres generaciones.

El aporte de la investigación a la generación de nuevo conocimiento sobre el tema en el ámbito internacional, corresponde a la oportunidad de integrar el conocimiento empírico en turismo rural de los habitantes de una localidad de la zona rural del corregimiento de Bruselas en el municipio de Pitalito Huila y el conocimiento científico de profesionales especializado en diferentes áreas afines al tema, sumando experiencias en turismo rural, desarrolladas con éxito en Colombia y en otras partes del mundo.

Además de lo anterior el nuevo conocimiento está asociado con el disfrute de lo local, lo tradicional, lo campesino; bien sea orientado hacia culturas diferentes o para propiciar un reencuentro con culturas propias. Los interesados en conocer y visitar sitios históricos tales como museos, senderos y parques arqueológicos aledaños, como también los que buscan conocer y compartir costumbres con otras sociedades, visitar lugares, consumir alimentos ancestrales y participar de las diferentes costumbres folclóricas, encontrarán en el desarrollo de esta investigación la oportunidad de un intercambio de conocimientos.

Colombia tiene una muy interesante experiencia para mostrar en la región. En el año 1991 prácticamente no existían desarrollos de turismo rural en el país. Las autoridades de uno de los más pequeños Departamentos, el Quindío, región tradicionalmente productora de café, decidieron impulsar el turismo rural a partir de las haciendas cafeteras. El éxito de la política es contundente, se pasó de cuatro haciendas en 1991, a las seiscientas actuales; y el Quindío se convirtió en la segunda región turística del país luego de Cartagena de Indias.

Para el logro del éxito señalado fue muy importante una política pública sostenida desde diferentes ámbitos, entre las cuales se pueden contar la promoción nacional a través del Ministerio de Comercio, Industria y Turismo; la promoción local por la Secretaría de Turismo del Departamento del Quindío; la formación profesional a través del Servicio Nacional de Aprendizaje (SENA), la institución nacional de formación para el trabajo y la política nacional que avanzó en brindar seguridad al turismo a partir del Programa "Vive Colombia. Viaja por ella".

La experiencia de turismo rural del SENA en el eje cafetero de Colombia nos cuenta que el turismo rural se desarrolla en un medio, el rural, que para el caso del Eje Cafetero (conformado por los Departamentos de Caldas, Quindío y Risaralda), con todas sus características de calidad y particularidad, se han convertido en la actualidad en un elemento clave y definitivo de la personalidad del propio destino. Es, además, la actividad agrícola, y particularmente el café, la que ha contribuido a crear la identidad rural del espacio, ayudando a mantener y conservar sus tradiciones 
(arquitectónicas, artesanales y culturales. Vertiginosamente se han venido desarrollando y fortaleciendo nuevas actividades en la región, como es el caso del turismo rural. A pesar del débil sistema económico y la demanda insatisfecha de personal especializado, cuenta a su favor con un particular potencial, el del patrimonio natural, histórico y cultural poco degradados, que se han convertido en ventajas comparativas en virtud de ser la mejor alternativa hoy frente a las actividades de ocio masivo y a los tradicionales productos de sol y playa. El Quindío, tiene además de las ya mencionadas riquezas del patrimonio social y de paisaje de la región, otros insumos más recientemente desarrollados: los parques temáticos; entre los que se cuentan:

Experiencias significativas son las Posadas Turísticas, iniciativa del Gobierno Nacional liderada por la Dirección de Turismo del Ministerio de Comercio, Industria y Turismo, donde participan también el Viceministerio de Vivienda a través de la Dirección de Vivienda del Ministerio de Ambiente, Vivienda y Desarrollo Territorial, y el Banco Agrario que hace parte del Ministerio de Agricultura y Desarrollo Rural. El programa contemplado dentro del Plan Nacional de Desarrollo, plantea la realización de viviendas productivas turísticas y cuyo objeto es crear condiciones para alojar turistas en las viviendas de los residentes en los destinos; especialmente en aquellas regiones que no disponen de planta turística de alojamiento desarrollada. Las Posadas Turísticas de Colombia son una novedosa alternativa de alojamiento creada para fomentar el turismo y generar posibilidades de ingreso a familias de bajos recursos económicos, en lugares del país que se destacan por su gran belleza, además de ser destinos con vocación y atractivos turísticos especiales, con riqueza natural y étnica. Así es como dicho programa permite que la mujer cumpla un rol diferente, se involucran todos los miembros del grupo familiar, se crean beneficios para la comunidad; en resumen, se está propiciando el desarrollo local y un mejoramiento de la calidad de vida, al mismo tiempo que se muestra otra cara del país.

Hablamos de construcciones que coexisten con el medio ambiente, diseñadas con calefactores de agua con energía solar, mejores sistemas de aprovechamiento del agua residual. Sus mujeres, como protagonistas locales y microempresarias, apoyan y promueven el desarrollo de su comunidad, los valores de la familia y la gastronomía local.

El problema está enmarcado por medio de motivación y capacitación en formulación de investigación inicialmente a los propietarios de predios rurales aptos para el desarrollo de la propuesta de investigación ubicada en la zona rural del corregimiento de Bruselas del municipio de Pitalito Huila.

La base teórica en que se sustenta el presente trabajo de investigación son los postulados de la teoría de desarrollo sostenible y sustentable y la teoría de las ventajas comparativas conocidas como el enfoque Heckscher - Ohlin.

La Teoría de desarrollo sostenible hace referencia al desarrollo que satisface las necesidades del presente sin comprometer la capacidad de las generaciones futuras de satisfacer sus propias necesidades. El Desarrollo sostenible se enfoca hacia la mejora de la calidad de vida de los habitantes de la tierra, sin aumentar el uso de recursos naturales más allá de la capacidad del ambiente de proporcionarlos. Se trata de tomar acciones, de cambiar políticas y prácticas en todos los niveles, desde el ámbito individual hasta el internacional.

\section{Objetivo general}

Elaborar una propuesta de desarrollo empresarial para permitir que el turismo sostenible sea una actividad conducente al desarrollo sostenible de la población rural en un territorio del corregimiento de Bruselas (Pitalito) para el año 2020.

\section{Objetivos específicos}

- Concientizar en los próximos 5 años por medio de sensibilización, motivación y capacitación al 90\% de los propietarios de las fincas en la importancia de nuevas actividades que generen desarrollo Rural autosostenible, sin comprometer los recursos de las generaciones futuras.

- Identificar las fincas y los sitios turísticos del corregimiento de Bruselas del Municipio de Pitalito.

- Realizar capacitaciones sobre investigación turística rural y diseño de página Web en el corregimiento de Bruselas del Municipio de Pitalito.

- Motivar a los participantes de la investigación "Turismo Rural" a conformar una unidad empresarial, legalmente constituida para la generación de empleo.

\section{Cómo se llevará a cabo la propuesta}

El proyecto de investigación será de corte descriptivo con aporte del enfoque empírico, analítico y en búsqueda de compresión, interpretación y caracterización del problema objeto de estudio y de esta forma plantear conclusiones con el fin de dar soluciones al Turismo Rural a los actores participantes en la comunidad del Corregimiento de Bruselas

Por las características del estudio, el diseño de la investigación será no experimental, de carácter transeccional ya que se realizará sin manipular deliberadamente las variables, observando los fenómenos tal y como se dan en su contexto natural, para después analizarlos y fue transeccional por cuanto los datos fueron recolectados en un tiempo único con el propósito de describir las variables en estudio y analizar su incidencia e interrelación en un momento dado. 
El diseño de la investigación no experimental transeccional descriptiva es aquella en la cual las variables independientes no son manipuladas y la interacción entre éstas se realiza sin intervención o influencia directa y son observadas tal y como se encuentran en su contexto natural; así mismo, los datos son recolectados en un solo momento, indagando la incidencia y las formas en que pueda manifestarse una o más variables. En conclusión, estos estudios presentan el estado de una variable en uno o más grupos o indicadores en un determinado momento

\section{Lista de Actividades}

- Visita a actores sociales del corregimiento de Bruselas interesados en formar parte de la investigación "Turismo rural" para definir necesidades, expectativas y metodología de trabajo.

- Visita y carta de solicitud al SENA para Definir alianza estratégica para lograr la consecución de capacitación y certificación en Guianza turística.

- Socialización de oferta que los propietarios de los predios están en capacidad de ofrecer al turista.

- Búsqueda de financiamiento por medio de Universidad, SENA, Red Empresarial, para llevar a cabo el desarrollo del investigación "Turismo Rural".

- Escoger un espacio adecuado para llevar a cabo las respectivas capacitaciones y certificaciones a realizar, teniendo en cuenta la adaptación de la escuela o lugar seleccionado.

- Motivación, socialización y toma de decisiones para conformar una unidad empresarial.

- Identificar que fincas están dentro del investigación y las opcionales para vincularse a él.

- Toma de videos y material fotográfico, socialización de diseño, recopilación de información necesaria, selección del diseño de la página web, diseño de rutero, para promocionar el turismo rural del corregimiento de Bruselas.

\section{Plan de gestión}

Las mancomunidades son instrumentos para una gestión desde lo local, con ámbitos territoriales diversos y amplios; no reemplazan a otras entidades de gobierno, ni tampoco se limitan al ámbito de una municipalidad, en cuya voluntad asociativa reposan el carácter de actores con alto grado de emprendimiento empresarial. Sin embargo, Sus investigación se institucionalizan y amplían en términos de dimensiones y de los actores de la actividad del Gobierno local, departamental nacional y mundial. Es por esto que es muy importante para el corregimiento de Bruselas vincular a esta actividad actores importantes como instituciones gubernamentales ONG y demás instituciones que apoyen el desarrollo empresarial turístico rural.

Teniendo en cuenta que los grupos de comunidades surgen de la voluntad local, y no se forman por mandato de ley, pero requieren de políticas públicas que las incentiven, especialmente para las zonas rurales y en pobreza, para afirmar el cambio territorial e institucional que pueden hacer posible su viabilidad. En los países donde existen normas de apoyo, las comunidades logran mejores resultados para los territorios donde se encuentran afianzando pasos estratégicos de calidad en la gestión territorial.

La estrategia a utilizar es mandar copia de los proyectas a todas las instituciones que posiblemente contribuyan a financiar el desarrollo de pequeñas comunidades locales, esto en aras de tocar puertas para buscar el financiamiento de investigación que buscan el desarrollo estratégico empresarial.

Al incorporar progresivamente a los diversos actores del territorio y asumir los desafíos en sus distintas dimensiones (físico-geográfico-técnico, de integración social, de identidad cultural, de vinculación y de la demarcación política administrativa), la mancomunidad se constituye en un espacio social de concertación para la acción conjunta y el adecuado tratamiento de intereses, donde puedan vincularse con diversas entidades internas $y$ externas sin limitarse a su afiliación política, sino poniendo por delante asuntos comunes orientados hacia el desarrollo local generando una gran oportunidad de abordar procesos de desarrollo económico desde una perspectiva no limitativa a la demarcación administrativa de servicios de los gobiernos locales, teniendo la posibilidad de generar investigación para ser financiados por otros gobiernos ( ONG ) articuladores que ayudan a superar la tendencia localista de la inversión administrativa.

Al igual se requiere una política que garantice su sostenibilidad, con recursos Propios y con medidas que incentiven su consolidación y respondan a las necesidades y aspiraciones de los actores de la mancomunidad local del corregimiento de Bruselas municipio de Pitalito. 
